\title{
Secondary ion mass spectrometry signatures for verifying declarations of fissile-material production
}

\author{
D. Willingham*, B. E. Naes, K. A. Burns and B. D. Reid \\ Pacific Northwest National Laboratory, 902 Battelle Boulevard, Richland, WA 99352 USA
}

\begin{abstract}
Direct analysis of uranium enrichment facility components were performed using secondary ion mass spectrometry (SIMS). A standard protocol was developed to enable preparation of SIMS samples from a corroded pipe piece without disturbing the corrosion layer. Unique uranium, oxygen and fluorine containing signatures were discovered in the corrosion layer by performing a mass scan of the region of interest from 230-280 amu. These signatures identified the source of the corrosion layer as uranium hexafluoride $\left(\mathrm{UF}_{6}\right)$ or an associated hydrolysis product. Isotopic analysis of the corrosion layer determined enrichment of ${ }^{235} \mathrm{U}$ to a value of $0.0116 \pm 0.0019$ for the ${ }^{235} \mathrm{U} /{ }^{238} \mathrm{U}$ isotopic ratio as compared to the NIST traceable standard (CRM 112-A) with a natural ${ }^{235} \mathrm{U} /{ }^{238} \mathrm{U}$ isotopic ratio of $0.007254 \pm 0.000004$. SIMS depth analysis revealed that the corrosion layer was isotopically homogenous to a depth of $\sim 23.5$ $\mu \mathrm{m}$. Optical profilometry measurements prior to and following SIMS depth analysis were used to determine a sputter rate of $0.48 \mathrm{~nm} / \mathrm{s}$ for $18.5 \mathrm{keV} \mathrm{O}^{-}$ion bombardment of the corrosion layer. The data presented is conclusive evidence that SIMS depth analysis can be used to identify novel nuclear archeology signatures from uranium enrichment components and perform meaningful isotopic analysis of these signatures.
\end{abstract}

Keywords Secondary ion mass spectrometry, Signatures, Isotope ratios, Uranium enrichment, Nuclear archeology

\section{Introduction}


The Nuclear Non-Proliferation Treaty (NPT) and other efforts to block the spread of nuclear weapons have created the need for a variety of verification technologies [1-5]. These methods aim to support the International Atomic Energy Agency's (IAEA) safeguards efforts in verifying declarations of fissile material production at declared facilities. This validation process, often termed nuclear archeology [6-8], is of great importance to the nonproliferation community responsible for monitoring a growing number of uranium enrichment facilities worldwide. The most difficult part of verifying special nuclear material production is obtaining an analytical signature that is not only indicative of the current enrichment campaign, but also seeks to characterize the complete history of prior cascade configurations. In most cases, acquisition of nuclear materials from within enrichment facilities is critical to treaty verification. Chemical compounds $\left(\mathrm{UF}_{6}, \mathrm{UO}_{2} \mathrm{~F}_{2}, \mathrm{HF}\right.$, etc.) used in uranium enrichment facilities are highly reactive and can corrode metal components (i.e. centrifuges, pipes, valves, etc.) $[9,10]$. Theoretically, corrosion layers on enrichment facility hardware contain unique isotopic signatures that can be used to establish operational history. Nuclear archeology aims to identify these signatures, accurately and precisely measure them and create computational models that translate the measured data into a record of the fissile-material production.

A robust measurement technique for determining the operational history of an enrichment facility does not currently exist; however, environmental sampling using swipe materials has been successfully used to determine current and past operations [11,12]. These microanalytical methods do not preserve spatial resolution from the sample surface, but do allow for the identification of rare particles that may be representative of historical operations or clandestine activity. A proposed methodology must meet several criteria in order to be effective. The method would ideally be a technique capable of high abundance sensitivity, atomic and 
molecular specificity and provide high precision and accurate isotopic data. Additionally, sample heterogeneity both spatially and in depth must be preserved to locate unique signatures. Similar signatures have previously been identified by SIMS analysis of swipe materials; however, this method does not preserve depth information. The methodology purposed herein aims to preserve the sample heterogeneity in depth and is, therefore, more ideal because signatures of interest are not diluted. This method is a way to access more information than was previously available with other SIMS techniques. Although many radiometric analysis techniques have proven to be useful in similar analyses, mass spectrometry is ideal for the identification and measurement of nuclear archeology signatures [6-8,13].

SIMS provides a useful method to exploit isotopic signatures resident in enrichment facility equipment and assists in determining an estimate of the enrichment levels and operational history. SIMS is a mass spectrometry technique that uses high energy primary ions to generate secondary ions indicative of the isotopic composition of solid samples. These secondary ions are extracted into a mass spectrometer where they are separated by their mass-tocharge ratios. In addition, SIMS has been used to provide the isotopic composition of a sample as a function of depth [14-16]. SIMS is, therefore, a powerful analytical method for uncovering embedded isotopic information via depth profiling. A SIMS-based technology for validating the enrichment production history of declared facilities could have valuable application for resolving international concerns associated with an ever-growing number of uranium enrichment facilities.

A possible scenario where SIMS could provide valuable information about the operational history of a declared enrichment facility is as follows. A hypothetical uranium enrichment facility was operated over 10 years, producing civilian sector nuclear fuel with a ${ }^{235} \mathrm{U}$ enrichment of $3 \%$. Over this duration, $\mathrm{UF}_{6}$ within the facility interacted with the metal 
components of the enrichment facility generating a corrosion layer primarily composed of uranyl fluoride $\left(\mathrm{UO}_{2} \mathrm{~F}_{2}\right)$ and uranium sub-fluorides $\left(\mathrm{UF}_{4}, \mathrm{U}_{2} \mathrm{~F}_{9}, \mathrm{UF}_{5}\right.$, etc.). The growth of this corrosion layer decreases exponentially from the time of the first exposure of the bare metal components to the $\mathrm{UF}_{6}$. After this initial period of civilian operation, the enrichment facility was reconfigured to run as a research reactor and operated for 6 months producing uranium with a ${ }^{235} \mathrm{U}$ enrichment of $20 \%$. The facility was subsequently reconfigured to its original state, once again, producing civilian sector nuclear fuel at $3 \%$ for an addition 5 years. Any metal components that were in contact with $\mathrm{UF}_{6}$ throughout these reconfiguration periods would have a corrosion layer mainly composed of uranium compounds with a ${ }^{235} \mathrm{U}$ enrichment of $3 \%$, but with a buried layer of corrosion products with a ${ }^{235} \mathrm{U}$ enrichment of $20 \%$. SIMS depth analysis of the corrosion layer could reveal an isotopic distribution first of ${ }^{235} \mathrm{U}$ enrichment of $3 \%$, then a thin layer of $20 \%{ }^{235} \mathrm{U}$ enrichment, then back to $3 \%$. In this way, SIMS depth analysis could be used to verify the enrichment production history of this declared facility accurately identifying the period of time for which the facility was operated as a research reactor. In addition to information about the sequence of different ${ }^{235} \mathrm{U}$ enrichment exposures, if the growth rate of the corrosion layer was well known and adequately characterized, specific information about the individual exposure times could be obtained.

\section{Experimental}

The enrichment facility component analyzed consisted of a stainless steel pipe that retained a layer of material (corrosion layer) on the inside surface; this internal portion of the pipe was exposed to an unknown set of nuclear processing conditions. Sub-samples were cautiously extracted from the pipe without disturbing the corrosion layer by drilling $1 / 3$ inch discs from the side, using a hole-saw (Fig. 1a). Because the pieces sampled from the pipe had a finite diameter, 
a slight curvature could be seen in the resulting sample. Although curved samples typically present difficulties for SIMS analysis, the curvature over the area of interest proved to be minimal. Moderate use of a mechanical file along the cut edges of the disc aided the reduction of burred edges and ensured a relatively flat surface for analysis when mounting. The sample discs were then mounted in a typical CAMECA SIMS sample holder, shown in Fig $1 \mathrm{~b}$ and entered into the vacuum chamber without further sample preparation.

Prior to SIMS analysis, radiometric counting was performed on the samples and no detectable uranium signals were measured. The radiometric counting measurements were taken with a $70 \%$ relative efficacy coaxial high purity germanium detector (HPGe) by Ortec. This detector is classified as low background; meaning that the materials used for construction are screened for radio-purity and the system is constructed in a manner to reduce background signals from the detector assembly itself. The detector is cooled with liquid nitrogen and cryostat controlled. The data from the detector was acquired using a Canberra Lynx digital signal processor and analyzed using the Canberra Genie2000 gamma analysis software suite.

SIMS analysis was performed on the upgraded CAMECA ims-4f at Pacific Northwest National Laboratory; a general description for the ims- $4 \mathrm{f}$ instrument can be found in detail elsewhere $[17,18]$. Briefly, the CAMECA ims-4f is a dynamic SIMS instrument that can be operated in both ion microscope (lower current, larger field-of-view) and microprobe (higher current, smaller field-of-view) modes. The SIMS analysis presented herein was performed with a duoplasmatron ion source generating $\mathrm{O}^{-}$ions at $12.5 \mathrm{kV}$. The secondary ions resulting from primary ion sputtering were accelerated with a $6 \mathrm{kV}$ positive bias on the sample, resulting in impact energy of $18.5 \mathrm{keV}$. Sputtered ions pass through an electrostatic analyzer and are then magnetically separated as a function of their mass-to-charge ratio; the secondary ion image field 
was $150 \mu \mathrm{m}$. The data collected for this report was obtained exclusively using an electron multiplier.

Pre- and post-SIMS analysis topographical measurements were performed by an optical profilometer (AEP Technology NanoMap 1000 wli 3D). These measurements were made by using a $150 \times 150 \mu \mathrm{m}$ field-of-view and medium $(\sim 1.0 \mu \mathrm{m})$ optical resolution. Analysis of the surface roughness was made prior to SIMS depth analyses to characterize the initial topography of the corrosion layer. Measurements of the SIMS depth analysis craters were performed following the isotopic measurements of the samples in order to provide information about the measured depth as well as the sputter rate of the SIMS depth analysis.

\section{Results and Discussion}

The initial SIMS analysis of the pipe piece focused on obtaining mass spectra from the corrosion layer in order to determine the presence of any unique nuclear archeology signatures. The mass spectra were obtained by scanning the magnet and measuring the ion counts as a function of mass-to-charge ratio. A representative mass spectrum of the corrosion layer is shown in Fig 2. This mass spectrum, representing a mass range from 230 to $280 \mathrm{amu}$, was selected to encompass $\mathrm{U}^{+}, \mathrm{UO}^{+}$and $\mathrm{UO}_{2}{ }^{+}$ions typically seen in analysis of uranium containing materials $[19,20]$. The first important observation is that the initial radiometric counting performed on this sample did not yield any detectable uranium signals, contrary to the SIMS analysis where ion signals from uranium isotopes $\left({ }^{235} \mathrm{U}^{+},{ }^{238} \mathrm{U}^{+}\right)$and uranium-containing compounds $\left(\mathrm{UO}^{+}, \mathrm{UF}^{+}, \mathrm{UO}_{2}^{+}\right.$and $\mathrm{UOF}^{+}$) were found. It is well known that $\mathrm{UF}_{6}$ reacts with various metals to form uranium subfluorides $\left(\mathrm{UF}_{4}, \mathrm{U}_{2} \mathrm{~F}_{9}, \mathrm{UF}_{5}\right.$, etc.) which then deposit as constituents of a corrosion layer [21,22]. Additionally, if exposed to water vapor, these sub-fluorides will hydrolyze to form hydrofluoric 
acid as well as oxygen and fluorine containing compounds such as uranyl fluoride $\left(\mathrm{UO}_{2} \mathrm{~F}_{2}\right)$ [23]. The mass spectrum in Fig 2 shows signatures that confirm two properties of the corrosion layer on the pipe piece: (1) the corrosion layer contains uranium and (2) the corrosion layer was likely created by exposure to $\mathrm{UF}_{6}$ or an associated hydrolysis product.

Data for the major isotopes of uranium $\left({ }^{235} \mathrm{U}\right.$ and $\left.{ }^{238} \mathrm{U}\right)$ were obtained from the pipe piece corrosion layer as a function of depth. The ion signals from the major uranium isotopes were monitored over a period of $\sim 13.5$ hours during the formation of a SIMS crater with an average ${ }^{235} \mathrm{U} /{ }^{238} \mathrm{U}$ ratio of $0.0116 \pm 0.0019$. Ignoring ${ }^{234} \mathrm{U}$ and ${ }^{236} \mathrm{U}$, which were not counted, the ${ }^{235} \mathrm{U}$ enrichment is $1.16 \pm 0.19$ percent. This value was corrected for mass fractionation by using a NIST traceable standard (CRM-112A) with a certified ${ }^{235} \mathrm{U} /{ }^{238} \mathrm{U}$ ratio of $0.00725 \pm 0.00004$ and an observed ${ }^{235} \mathrm{U} /{ }^{238} \mathrm{U}$ ratio of $0.00789 \pm 0.00013$ (Appendix). Unfortunately, the ${ }^{235} \mathrm{U} /{ }^{238} \mathrm{U}$ ratio of the material produced in the enrichment facility is unknown and, therefore, a direct comparison cannot be made. Fig 3 illustrates the data taken as the ${ }^{235} \mathrm{U} /{ }^{238} \mathrm{U}$ ratio as a function of total depth in $\mu \mathrm{m}$. The scatter in the data is largely counting statistics limited and therefore more uncertainty exists for lower count rates than for higher count rates (i.e. as the signal decreases with depth there is more uncertainty in the measured ${ }^{235} \mathrm{U} /{ }^{238} \mathrm{U}$ isotope ratio). The data in Fig 3 verifies two important parameters of the corrosion layer: (1) it is generated by a uranium fluoride compound enriched in ${ }^{235} \mathrm{U}$ above the natural ${ }^{235} \mathrm{U} /{ }^{238} \mathrm{U}$ isotopic ratio and (2) it is isotopically homogenous in depth over the course of the analysis (substrate signals were not observed). In addition, although the corrosion layer was sputtered for $>10$ hours, expected (Cr, $\mathrm{Ni}$ and/or Fe) signals from the stainless steel substrate were not observed. This indicates that either the sputtering rate for the corrosion layer is significantly slower or that the corrosion layer is significantly thicker than originally hypothesized. It must be noted that, assuming a constant 
and surplus supply of parent $\mathrm{UF}_{6}$, the excess observed thickness of uranyl fluoride and uranium sub-fluoride compounds could, in fact, be indicative of the diffusion limited growth of a passivation layer. This growth would be asymptotic in nature eventually resulting in complete passivation of the surface of the pipe piece. Further electron microprobe analysis (EMPA) or analogous cross-sectioning technique would be needed to confirm the thickness of this passivation layer.

An understanding of the SIMS depth analysis relies not only on a complete characterization of the composition of the corrosion layer, but is also reliant on the rate at which the corrosion layer material can be removed. Optical profilometry measurements of the corrosion layer are shown in Fig 4 before (a) and after (b) SIMS depth analysis. Analysis of the corrosion layer prior to SIMS depth analysis reveals a rough surface with a root mean squared (RMS) roughness of $2.84 \mu \mathrm{m}$. This observation can be explained by the nature of the formation of the corrosion layer itself. Previous work has shown that $\mathrm{UF}_{6}$ corrosion of metal initiates at surface defects and then proceeds by an exponential decay growth mechanism: rapid film growth followed by an exponential decrease to virtually zero film growth at $t_{\infty}$ [9]. The formation of a rough corrosion layer primarily arises from multiple initiation sites separated laterally and as a product of time as well. Additionally, it is likely that the corrosion layer thickness is variable over the internal surface of the pipe piece and should be taken as an average over several SIMS depth profile measurements rather than a finite number from a single location. A total of three replicate SIMS depth analyses were performed with a representative SIMS depth analysis crater shown in Fig 4b (isotopic data from this crater analysis is shown in Fig. 3) with an area of $\sim 100$ $\mu \mathrm{m}^{2}$ and a depth of $\sim 23.5 \mu \mathrm{m}$. A sputter rate for this analysis, expressed as the depth of sample reached per second of SIMS depth analysis, was calculated as $0.48 \mathrm{~nm} / \mathrm{s}$; sputter rates from the 
other two analyses were not measured. Sputter rates typically vary between 0.5 and $5 \mathrm{~nm} / \mathrm{s}$ and are dependent on primary ion beam intensity, sample material, and crystal orientation [24]. Although the SIMS depth profile did not reach the corrosion layer/metal interface, the sputter rate is a useful parameter for determining the ultimate SIMS capabilities for future analysis of enrichment facility components. The optical profilometry measurements confirm that the observed corrosion layer is tens of micrometers thick, but also dictates that the $18.5 \mathrm{keV} \mathrm{O}^{-}$ sputter rate for the corrosion layer is relatively low. Future efforts may result in employing methods for producing cross-sections for given corrosion layers $[25,26]$, which have been demonstrated herein to be time prohibitive for direct analysis by SIMS depth profiling. A method of particular interest is to use the micromachining abilities of a focused ion beam (FIB) to cut, extract, move and weld a very small section of the enrichment facility component of interest for subsequent SIMS analysis. This technique translates the depth information in the zaxis into a 2D image in the $x-y$ axes, thereby allowing the analysis of much thicker corrosion layers.

\section{Conclusion}

For the first time, samples from a pipe extracted from a uranium enrichment facility were directly analyzed by SIMS with little to no sample preparation outside the sub-sampling approach outlined herein. Initial mass spectra of a piece of the pipe revealed that the corrosion layer present on the internal wall of the pipe did contain uranium and uranium compounds despite standard radiometric screening methods not detecting a measureable signal. The characteristic ions in the mass spectra verified the presence of oxygen and fluorine containing uranium molecules, which indicates that the corrosion layer was formed by $\mathrm{UF}_{6}$ or an associated hydrolysis product. The isotopic distribution of the corrosion layer was found to be enriched in 
${ }^{235} \mathrm{U}$ by comparison to natural uranium isotope abundances. The reported value was confirmed by the measurement of a NIST traceable standard (CRM 112-A). SIMS depth analysis showed the corrosion layer to be isotopically homogeneous to a depth of $\sim 23.5 \mu \mathrm{m}$. The corrosion layer/metal interface was not observed even after sputtering for $\sim 13.5$ hours, at a sputter rate of $0.48 \mathrm{~nm} / \mathrm{s}$. The preliminary results presented in this study represent a proof-of-principle that SIMS can be used to identify unique nuclear archeology signatures and measure the isotopic distribution of these signatures from uranium enrichment facility components.

Acknowledgements The research described in this paper was conducted under the Laboratory Directed Research and Development Program at Pacific Northwest National Laboratory, a multiprogram national laboratory operated by Battelle for the U.S. Department of Energy. 


\section{References}

[1] A. Gavron, Applied radiation and isotopes : including data, instrumentation and methods for use in agriculture, industry and medicine 63 (2005) 607.

[2] O.N. Lyakhova, S.N. Lukashenko, S.I. Mulgin, S.V. Zhdanov, Journal of environmental radioactivity 124 (2013) 13.

[3] H.S. Miley, C.E. Aalseth, T.W. Bowyer, J.E. Fast, J.C. Hayes, E.W. Hoppe, T.W. Hossbach, M.E. Keillor, J.D. Kephart, J.I. McIntyre, A. Seifert, Applied radiation and isotopes : including data, instrumentation and methods for use in agriculture, industry and medicine 67 (2009) 746.

[4] M. Schwaiger, F. Steger, T. Schroettner, C. Schmitzer, Applied radiation and isotopes : including data, instrumentation and methods for use in agriculture, industry and medicine 56 (2002) 375.

[5] T.J. Stocki, X. Blanchard, R. D'Amours, R.K. Ungar, J.P. Fontaine, M. Sohier, M. Bean, T. Taffary, J. Racine, B.L. Tracy, G. Brachet, M. Jean, D. Meyerhof, Journal of environmental radioactivity 80 (2005) 305.

[6] S. Fetter, Science \& Global Security 3 (1993) 237.

[7] J.M. Schwantes, M. Douglas, S.E. Bonde, J.D. Briggs, O.T. Farmer, L.R. Greenwood, E.A. Lepel, C.R. Orton, J.F. Wacker, A.T. Luksic, Anal. Chem. 81 (2009) 1297.

[8] T.W. Wood, B.D. Reid, C.M. Toomey, K. Krishnaswami, K.A. Burns, L.O. Casazza, D. S. Daly, L. L. Duckworth, Science \& Global Security 22 (2014) 4.

[9] J.M. Saniger, F. Alba, S.J. Garrido, E. Avendano, Corrosion Sci. 30 (1990) 903.

[10] S.C.P. Wang, C. Collins, S. Anghaie, E.D. Whitney, Nucl. Technol. 93 (1991) 399.

[11] Y. Ranebo, P.M.L. Hedberg, M.J. Whitehouse, K. Ingeneri, S. Littmann, J Anal Atom Spectrom 24 (2009) 277.

[12] G. Tamborini, Microchim Acta 145 (2004) 237.

[13] T.W. Wood, B.D. Reid, J.L. Smoot, J.L. Fuller, The Nonproliferation Review 9 (2002) 126.

[14] B. Marchand, N. Moncoffre, Y. Pipon, N. Bererd, C. Garnier, L. Raimbault, P. Sainsot, T. Epicier, C. Delafoy, M. Fraczkiewicz, C. Gaillard, N. Toulhoat, A. Perrat-Mabilon, C. Peaucelle, J. Nucl. Mater. 440 (2013) 562.

[15] E. Pizzi, P. Garcia, G. Carlot, H. Palancher, S. Maillard, B. Pasquet, I. Roure, C. Pozo, C. Maurice, in: I. Bezverkhyy, S. Chevalier, O. Politano (Eds.), Diffusion in Materials - Dimat 2011, Trans Tech Publications Ltd, Stafa-Zurich, 2012, p. 197.

[16] Y. Ranebo, M. Eriksson, G. Tamborini, N. Niagolova, O. Bildstein, M. Betti, Microscopy and microanalysis : the official journal of Microscopy Society of America, Microbeam Analysis Society, Microscopical Society of Canada 13 (2007) 179.

[17] M. Meuris, P. Debisschop, J.F. Leclair, W. Vandervorst, Surf Interface Anal 14 (1989) 739.

[18] H.N. Migeon, M. Schuhmacher, J.J. Legoux, B. Rasser, Fresen Z Anal Chem 333 (1989) 333.

[19] L. Desgranges, B. Pasquet, I. Roure, Appl. Surf. Sci. 257 (2011) 6208.

[20] Y.J. Zhu, N. Olson, T.P. Beebe, Jr., Environmental science \& technology 35 (2001) 3113.

[21] J.K. Gibson, R.G. Haire, Journal of Alloys and Compounds 181 (1992) 23.

[22] S.W. Vorster, F.P.A. Robinson, British Corrosion Journal 27 (1992) 151.

[23] F.E.T.K. D. Heymann, Anti-Corrosion Methods and Materials 5 (1958) 148.

[24] J.E. deVries, J. of Materi Eng and Perform 7 (1998) 303.

[25] C. Eriksson, P. Malmberg, H. Nygren, Rapid communications in mass spectrometry : RCM 22 (2008) 943.

[26] R. Kiebach, K. Norrman, C. Chatzichristodoulou, M. Chen, X. Sun, S.D. Ebbesen, M.B. Mogensen, P.V. Hendriksen, Dalton transactions (2014). 


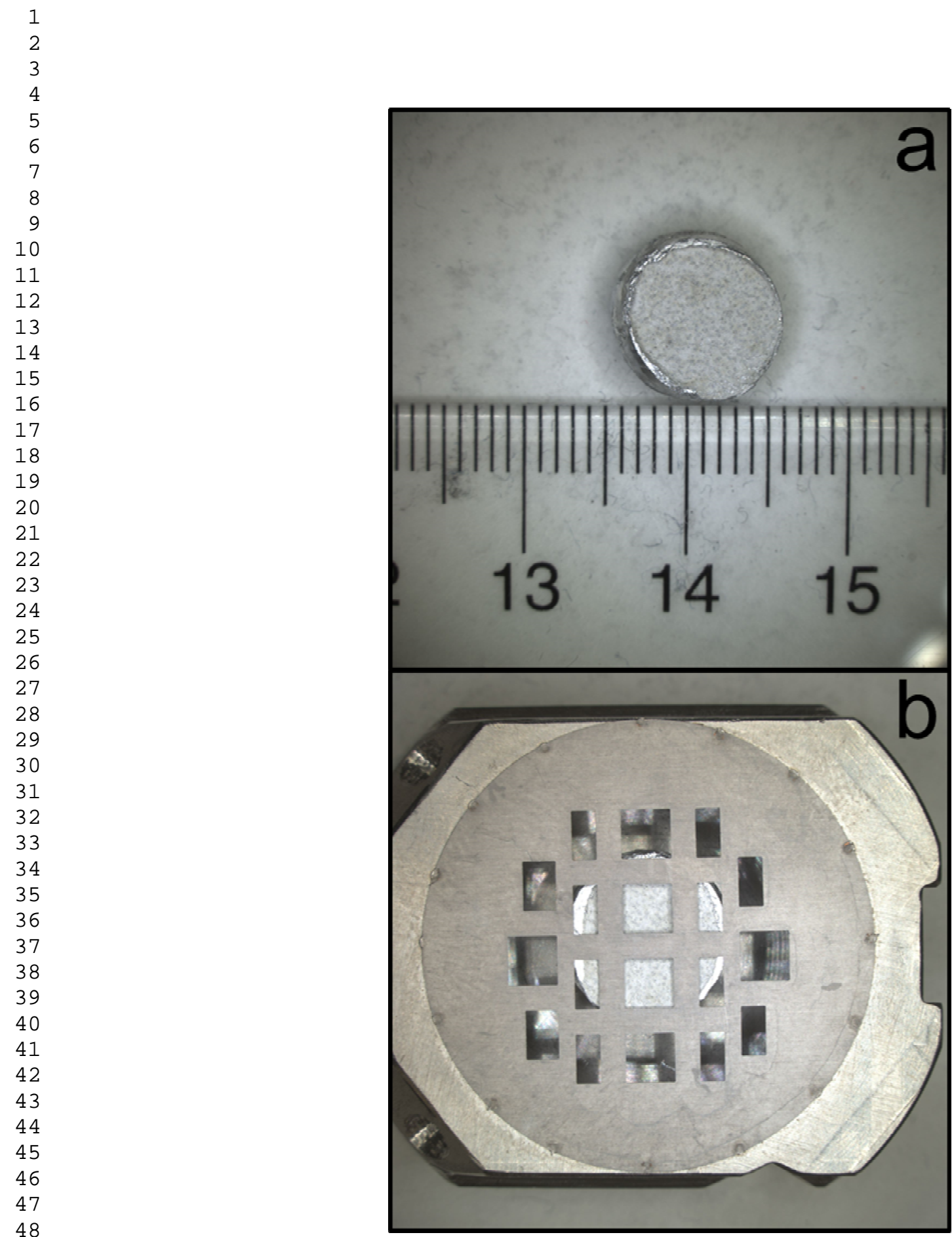

Fig 1. Circular disc section of the pipe sub-sample prepared for SIMS mounting (a) with a metric ruler for scale. The disc section (b) spring mounted in a standard CAMECA SIMS holder. The slight curve of the sample prevents the surface of the corrosion layer from contacting the back surface of the SIMS mount. 


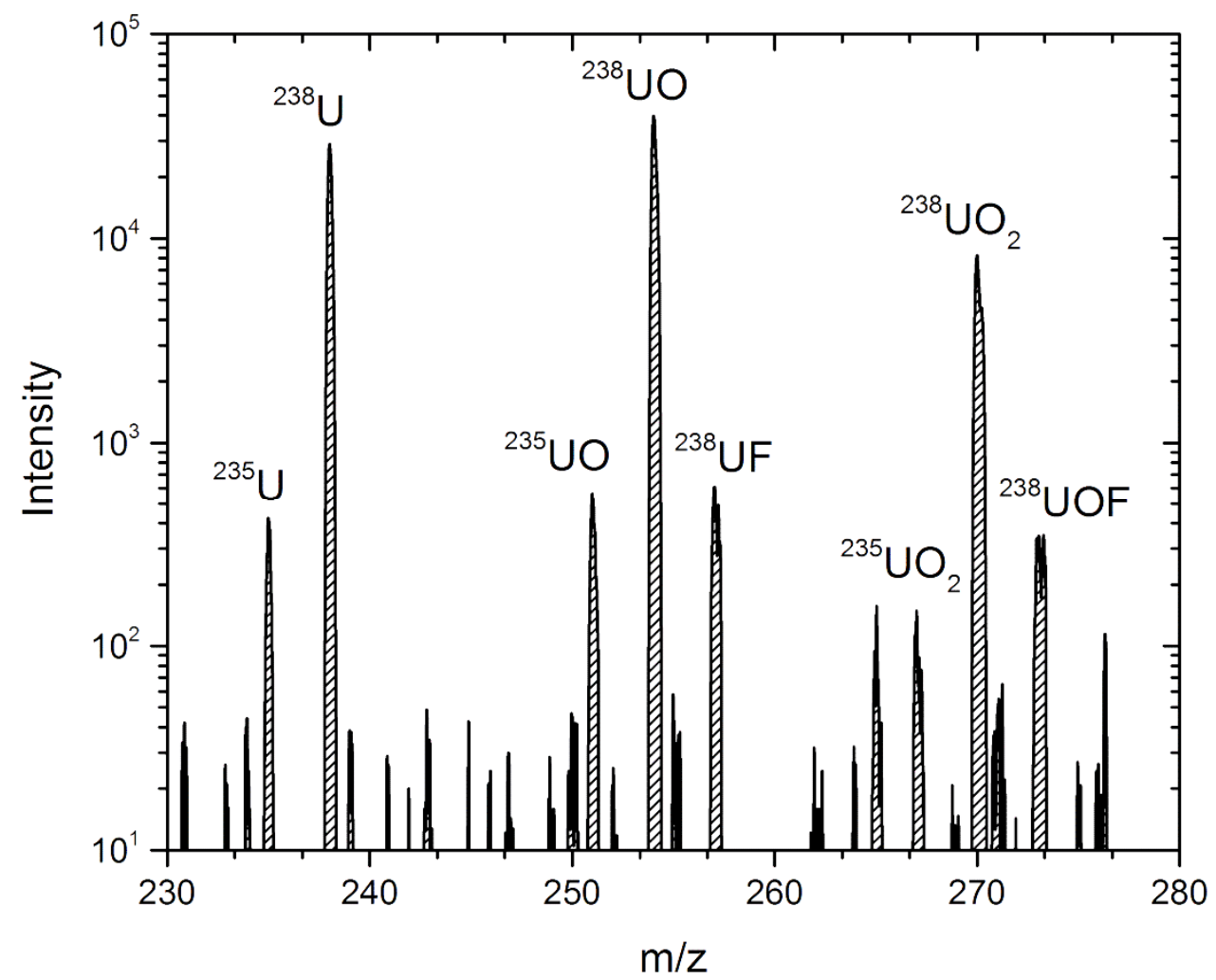

Fig 2. A representative mass spectrum of the corrosion layer is shown plotting the signal intensity as a function of the mass-to-charge ratio for the range 230 to $280 \mathrm{amu}$. The major ions have been identified as ${ }^{235} \mathrm{U}^{+},{ }^{238} \mathrm{U}^{+},{ }^{235} \mathrm{UO}^{+},{ }^{238} \mathrm{UO}^{+},{ }^{238} \mathrm{UF}^{+},{ }^{235} \mathrm{UO}_{2}{ }^{+},{ }^{238} \mathrm{UO}_{2}{ }^{+}$and ${ }^{238} \mathrm{UOF}^{+}$. Minor uranium isotopes and less abundant uranium molecular ions are present, but could not be positively identified. 
Fig 3. Summary of points measured throughout a representative SIMS depth analysis shown by plotting the ${ }^{235} \mathrm{U} /{ }^{238} \mathrm{U}$ isotope ratio as a function depth. The ${ }^{235} \mathrm{U} /{ }^{238} \mathrm{U}$ isotope ratio of $0.0116 \pm$ 0.0019 is represented by the mean (dashed line) and the standard deviation (solid lines).

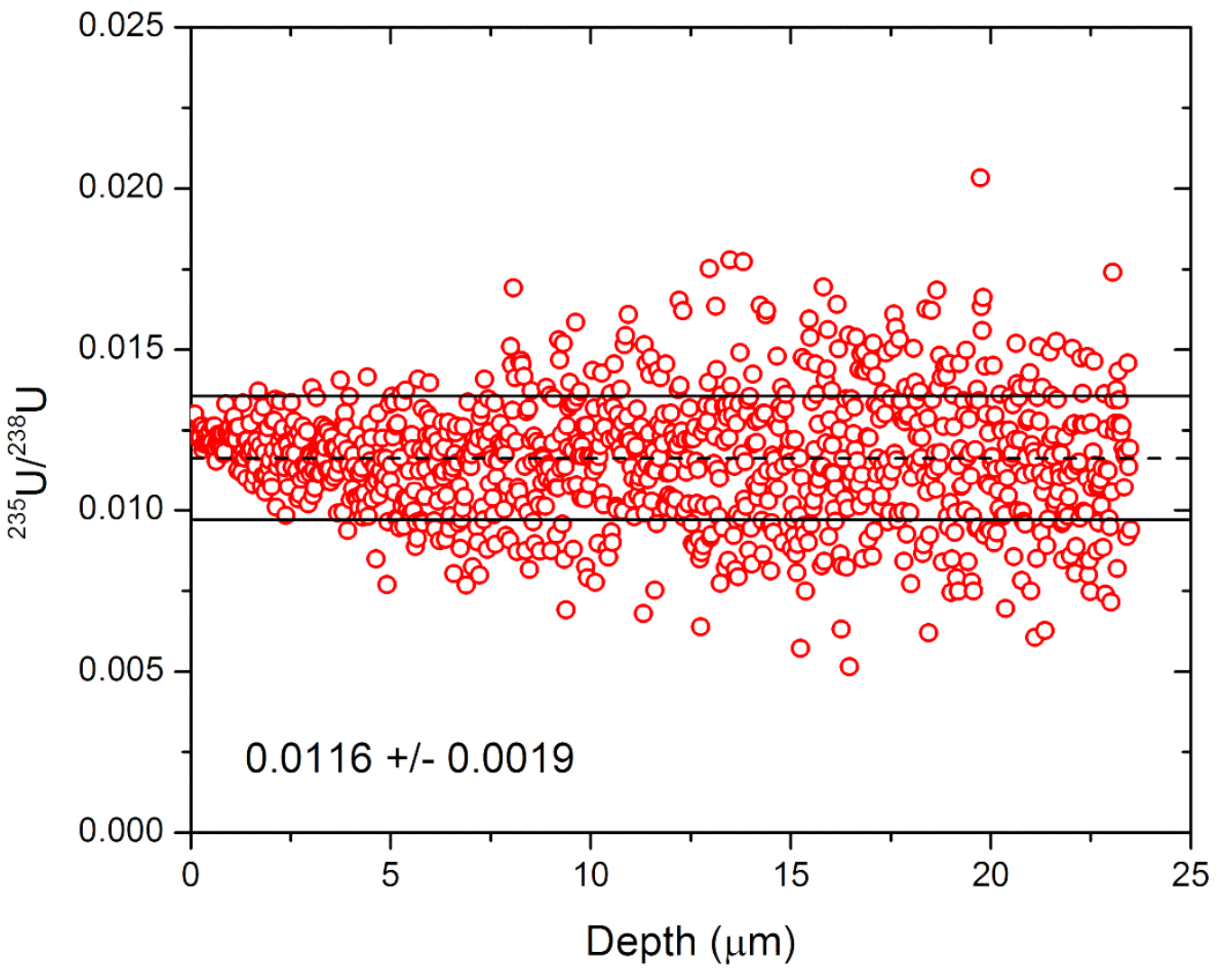



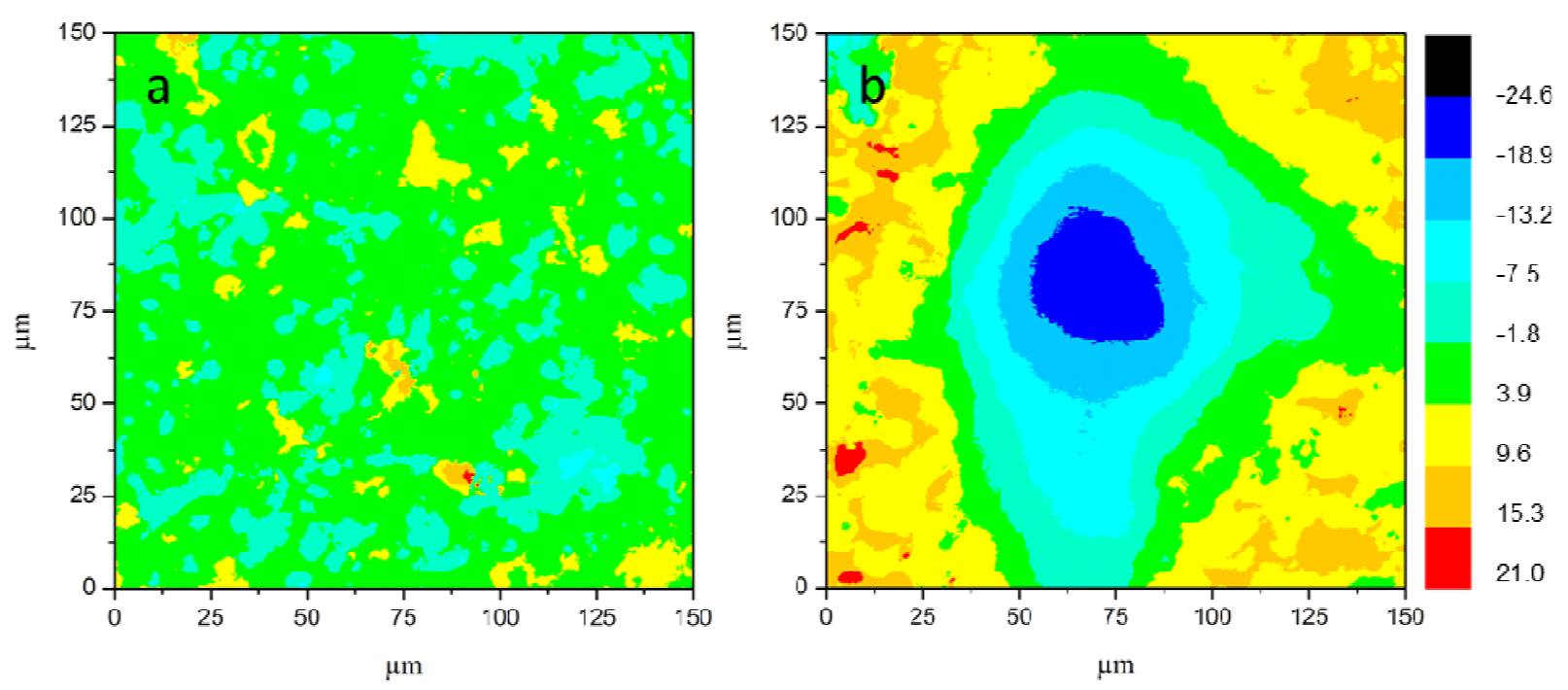

Fig 4. $2 \mathrm{D}$ representation of the optical profilometry measurements of the corrosion layer (a) prior to and (b) following SIMS depth analysis. Each square has a field-of-view of $150 \times 150$ $\mu \mathrm{m}$. The depth scale $(\mu \mathrm{m})$ is shown for deep regions in blue and surface regions in red. The scale applies to both images. 